\title{
Analysis of Properties Characteristic for Acoustic Emission Signals Recorded On-Line in Power Oil Transformers
}

\author{
F. Witos*, A. Olszewska And G. Szerszeń \\ Department of Optoelectronics, Silesian University of Technology, Krzywoustego 2, 44-100 Gliwice, Poland \\ Investigation results of properties characteristic for acoustic emission signals recorded in two selected power \\ oil transformers are presented. Signals were put to the filtration, whereas components coming from partial \\ discharges have been left. The calculations concerned: phase-time characteristics, averaging phase characteristics, \\ averaging short time Fourier transform spectrograms, amplitude distributions of signals, values of acoustic \\ emission descriptor with acronym ADC. On the ground of calculated basic characteristics and maps of ADC \\ descriptor three areas have been selected on lateral walls of transformer tanks. Acoustic emission signals recorded \\ in these areas were analyzed from the point of view how is influence of propagation path on these properties.
}

PACS: 52.80.-s, 77.22.Jp, 84.70.+p, 43.40.Le, 43.60.Lq

\section{Introduction}

Investigation of properties characteristic for acoustic emission (AE) signals recorded in power oil transformers is a very large and complex problem which has both cognitive and practical aspect.

Author's method used to analyze AE signals [1-6], applied to signals recorded in power oil transformers, enables us to elaborate the method how to investigate AE signals in power oil transformers which includes successively: recording of signals, calculation of amplitude distributions of signals, calculation of defined AE descriptors, working up of descriptor maps on lateral walls of transformer tanks, location of AE sources according to these maps by means of the method of maximum loudness (modified by the authors), and analysis of properties characteristic for signals recorded at points lying in the vicinity of identified sources. Presented stages of analysis are realized repeatedly within different frequency bands which enable to distinguish signals coming from different types of sources such as partial discharges (PD), Barkhausen's effect, oil circulation, and outer acoustic interferences.

At present descriptor maps are worked out on lateral walls of transformer tanks whereas real sources are situated in the whole volume of a transformer. This means that proposed method should be extended to consider that every power oil transformers are very complex objects also from the acoustic point of view. Such a feature results in complex path of elastic waves coming from a source (where impulses are generated) to a sensor (situated on outer surface of the transformer tank) which detects a signal.

This article is a contribution relating to working out of expanded methodology used to investigations of AE sig-

* corresponding author; e-mail: Franciszek.Witos@polsl.pl nals in power oil transformers which leads to elaboration of descriptor maps on lateral walls of transformer tanks, location of sources in the whole volume of a transformer, and analysis of properties characteristic for AE signals. The subject of the article are properties of AE signals recorded at a few measuring points lying in the vicinity of three different sources and analyzed in the band of $110-200 \mathrm{kHz}$, i.e. in the band where signals coming from partial discharges are dominant.

In order to investigate transformers an author's measuring system called AE DEMA-COMP [1-6] was applied; such a system has been equipped with AE sensors D9241A type, produced by Euro Physical Acoustic Corporation. Investigations concern two oil power transformers with transformer ratio of $120 / 20 / 6 \mathrm{kV}$ and rated power of 25 MVA. Transformers were of identical construction but different technical state resulting from their operation. AE signals have been recorded at chosen measuring points, accessible for measurement. These points create a measuring network on lateral wall surfaces of transformer tanks. Signals were recorded several times at each measuring point; such a recording lasted by $2 \mathrm{~s}$ (100 periods of the supply voltage). Measurements were realized on-line in power stations during operation of each transformer.

Within literature frequency bands suitable for observation of partial discharges in transformers by means of AE method are following: $70-180 \mathrm{kHz}$ [8-10], 100-200 $\mathrm{kHz}$ [12], up to $500 \mathrm{kHz}[11,13,14]$ or more precisely up to $270 \mathrm{kHz}$ with a few bands [15].

\section{Maps of descriptors}

Recorded AE signals have been put to filtration within the band of $110-200 \mathrm{kHz}$, and next basic characteristics, amplitude distributions and AE descriptor with an acronym ADC [1-5] were worked up. Basing on kriging method, the descriptor maps on lateral walls of trans- 
former tanks have been worked and local maxima of descriptors have been found.

Maps of ADC descriptor worked up for analyzed frequency band are presented in Fig. 1a (for transformer 1) and in Fig. 1b (for transformer 2). The $X Y$ plane on these maps is a developed lateral wall of the transformer tank. Coordinates of the points on this plane are in centimeters and determined by the following way: values of $Y$ coordinates are calculated from the bottom of the tank, $X$ value is zero for the center of the tap changer, values of $X$ coordinates increase in direction of higher voltage side (high-voltage winding), values of $X$ coordinates decrease in direction of lower voltage side (low-voltage winding), maximum and minimum values of $X$ coordinates describe points opposite to points describing the center of the tap changer. Izolines at the maps connect points where calculated values of descriptors are the same. The darker places on the map the greater descriptor value and similarly - the greater degree of advance of AE signal recorded at a measuring point.
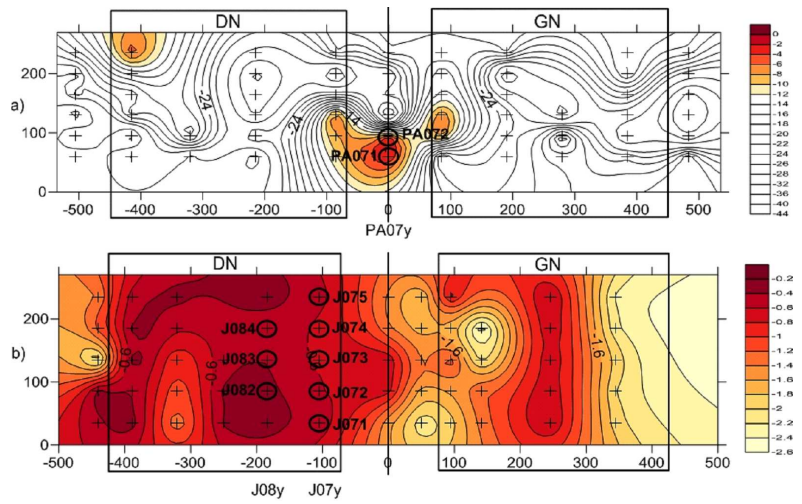

Fig. 1. Map of ADC descriptor worked up after analysis of recorded AE signals within the band of 110 $200 \mathrm{kHz}$ for transformer 1 (a) and transformer 2 (b). Dimensions of the tank $(X, Y)$ in centimeters: $X$ running position along the transformer tank, 0 - the center of the tap changer, positive $X$ values - part of the tank from higher voltage side, negative $X$ values part of the tank from lower voltage side, $Y$ - running height on the transformer tank, PA07y, J07y, J08j chosen measuring points lying at suitable columns and rows marked by $y$.

Positions of measuring points in the measuring network are also marked on the maps. Assumed symbols give in turn: name of the transformer (transformer 1 as PA, transformer 2 as J), number of the column and number of the row (rows are numbered by a growing way, counting from the top), for example PA075, J073.

\section{Analysis of properties characteristic for AE signals}

Properties of AE signals recorded at chosen measuring points are analyzed. The measuring points are situated in three areas: a) two measuring points situated in the area of the tap changer of the transformer 1: PA072 and PA071 (Fig. 1a),

b) measuring points situated in the column 7 for the transformer 2: J071, J072, J073, J074 and J075 (Fig. 1b),

c) chosen measuring points in the column 8 for the transformer 2: J082, J083 and J084 (Fig. 1b).

Characteristics belonging to chosen basic description of signals recorded in the area (a) of the transformer 1 are presented in Figs. 2 and 3. The signal recorded at PA072 point has the following properties: its main frequency bands are $110-130 \mathrm{kHz}$ and $140-150 \mathrm{kHz}$, it appears twice in duration of one period of the supply voltage in phases whose are different by $180^{\circ}$ and is very good located in the phase, "narrow" tunnels in the phase-time characteristic (Fig. 2a) testifies its periodical character. The signal recorded at PA071 point has the following properties: its main frequency bands are $110-130 \mathrm{kHz}$ and $140-150 \mathrm{kHz}$, within the signal there are two additional structures which appear twice during one period of the supply voltage in the phases which differ by $180^{\circ}$ (whereas phase ranges for these structures are considerably wider). The complex structure of the signal gives many tunnels in the phase-time characteristic (Fig. 3a). Phase distances between structures common for Figs. 2b, $3 \mathrm{~b}$ and additional structures appearing in Fig. $3 \mathrm{~b}$ are $20^{\circ}$ and $110^{\circ}$, respectively. Appraising the speed of the wave in the oil on about $1200 \mathrm{~m} / \mathrm{s}$ [7], it results from intervals between signals in averaging phase characteristic (Fig. 3b) that difference of propagation path should be a rank of $1.3 \mathrm{~m}$ and above $7 \mathrm{~m}$. Characteristics presented in Figs. 2 and 3 enable us to state that: PA072 point is situated across the source, to this point there arrives only AE wave running directly from the source whereas to PA071 point many waves arrive. Comparing distances of sources lying in the vicinity of the measuring point PA071 (Fig. 1a) and calculated difference of propagation paths $(1.3 \mathrm{~m}$ and $7 \mathrm{~m})$ it is visible that signals recorded at this point come from $\mathrm{AE}$ waves which are complex propagation path. Thus, even at neighbouring points of the transformer one can obtain very different structures of recorded signal which result from real propagation path $\mathrm{AE}$ impulses generated by $\mathrm{AE}$ sources and recorded at a given measuring point.

Characteristics belonging to a chosen basic description of signals recorded in the area (b) of the transformer 2 are presented in Fig. 4. We will analyze property of the structure (appearing for phases $175^{\circ}-180^{\circ}$ of the supply voltage) distinguished in Fig. 4a within the signal recorded at the measuring point J073. In the case of signals recorded at successive measuring points such a structure moves in direction of larger phases; at measuring point J071 it appears for $185^{\circ}-190^{\circ}$. Re-count of difference of phases for signals recorded at points J073 and J075 into difference of propagation paths of $\mathrm{AE}$ waves gives the value about 


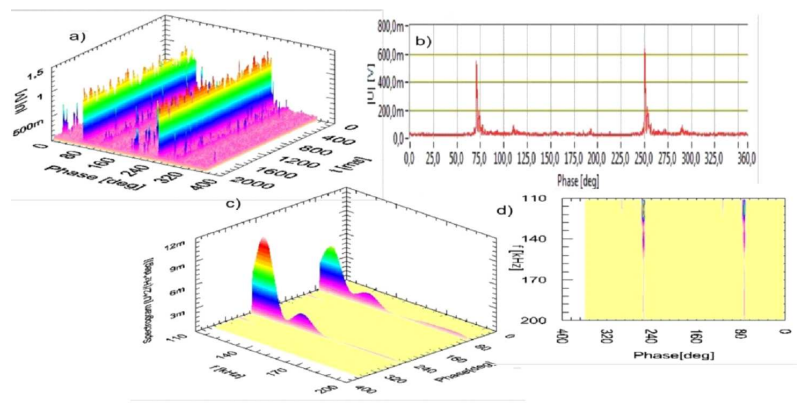

Fig. 2. Basic description of AE signal recorded at chosen measuring point of the transformer 1 (PA072) with filtration in the band of $110-200 \mathrm{kHz}$ : (a) phasetime characteristic, (b) averaging phase characteristic, (c) and (d) short-time Fourier transform (STFT) spectrograms, $\mathrm{ADC}=-2.66$.

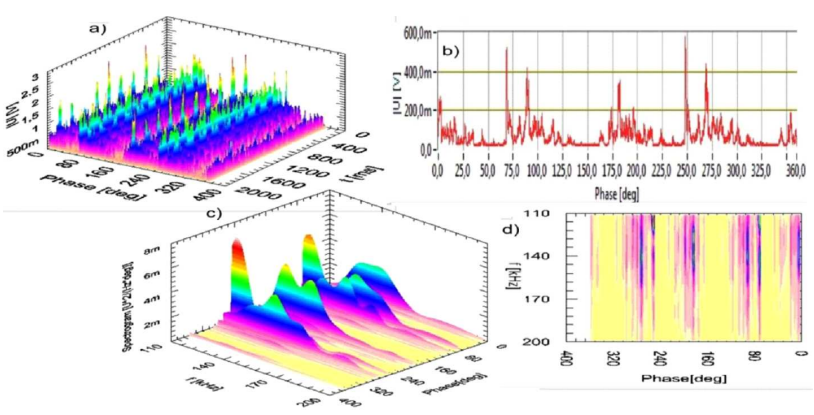

Fig. 3. Basic description of AE signal recorded at chosen measuring point of the transformer 1 (PA071) with filtration in the band of $110-200 \mathrm{kHz}$ : (a) phase-time characteristic, (b) averaging phase characteristic, (c) and (d) STFT spectrograms, ADC $=-1.86$.

$1 \mathrm{~m}$; it is worth adding that distance between measuring points J073 and J071 is $100 \mathrm{~cm}$. In turn, the signal at measuring point J073 in averaging STFT spectrograms (Fig. 4b) has a large frequency band; this band is narrowed down when goes away from the point J073 at moves in direction of lower frequencies. Collation of two above facts leads to a conclusion that sources coming from partial discharges appear in the vicinity of the measuring point J073; this signal spreads in considered area without obstacles and appears at other measuring points with delay resulting from the passable path but transformer oil damps more higher harmonics in the signal. Measuring points J071-5 are situated actually in the "empty" area of the transformer, i.e. between windings of L1 phase and the tap changer.

Characteristics belonging to a chosen basic description of signals recorded in the area (c) of the transformer 2 are presented in Figs. 5-7 where measuring points J082 and J083 are situated in the area of a vast source whereas the point J084 is out of the area of the source. According to Fig. $5 \mathrm{~b}$ and $6 \mathrm{~b}$, signals in the area of the source appear repeatedly in turn, twice during one period of the supply voltage and always in the same phase. This means that

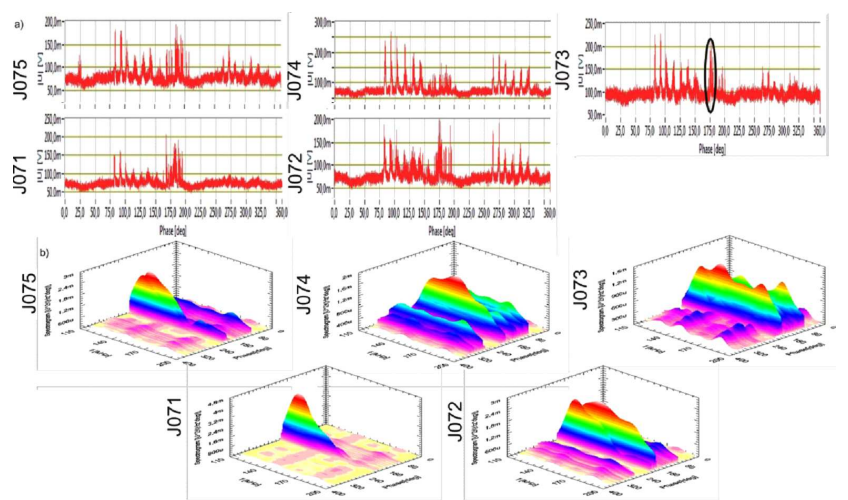

Fig. 4. Basic description of AE signal recorded at chosen measuring points of the transformer 2 (J07y, $y=1$, 2, 3, 4, and 5) within the band of 110-200 kHz: (a) averaging phase characteristic, (b) averaging STFT spectrograms.

partial discharges rise, last, and fade away (this process includes one peak in an averaging phase characteristic), and after some time - when conditions to initiate locally partial discharges in a PD source are met again - AE impulses are generated repeatedly. Such a mechanism is responsible for multiple peaks in an averaging phase characteristic. Since peaks in averaging characteristics for the both measuring points appear always in the same phase, particular elements of a vast source generate AE impulses simultaneously and the source has the same character in the whole area. At measuring point J084, situated belong the source area, there are AE waves spreads from the source to the measuring point, and submitted dumping processes (Fig. 7).

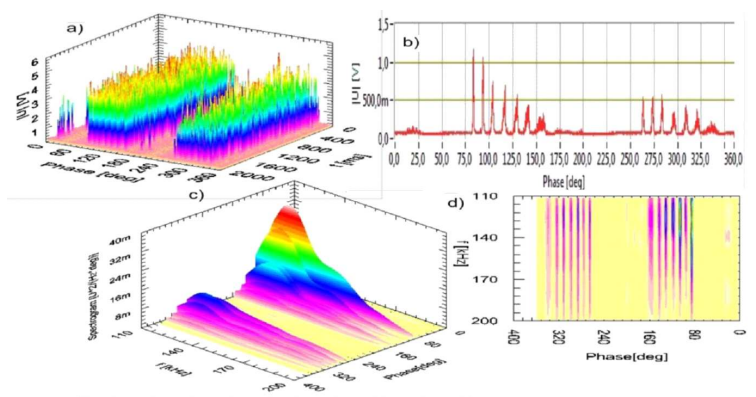

Fig. 5. Basic description of AE signal recorded at chosen measuring point of the transformer 2 (J082) within the band of 110-200 kHz: (a) phase-time characteristic, (b) averaging phase characteristic, (c) and (d) averaging STFT spectrograms, ADC $=-0.37$.

\section{Recapitulation}

AE method has been applied in order to research into AE signals coming from different acoustic sources in two selected power oil transformers. Such a distinction obtained in result of filtration of recorded signals within 


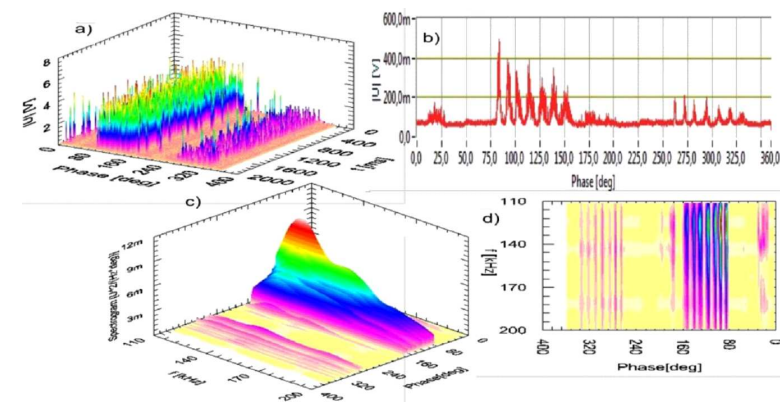

Fig. 6. Basic description of AE signal recorded at chosen measuring point of the transformer 2 (J083) within the band of 110-200 kHz: (a) phase-time characteristic, (b) averaging phase characteristic, (c) and (d) averaging STFT spectrograms, $\mathrm{ADC}=-0.41$.

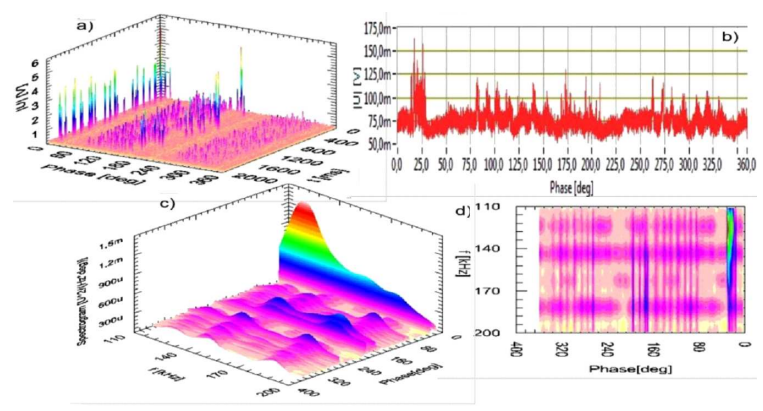

Fig. 7. Basic description of AE signal recorded at chosen measuring point of the transformer 2 (J084) within the band of 110-200 kHz: (a) phase-time characteristic, (b) averaging phase characteristic, (c) and (d) averaging STFT spectrograms, $\mathrm{ADC}=-0.55$.

the band of $110-200 \mathrm{kHz}$ i.e. in the band where signals coming from partial discharges are dominant.

After filtration, amplitude distributions of signals and values of defined AE descriptors with acronym ADC have been calculated. Those enabled us to work out descriptor maps on lateral walls of transformer tanks.

The following basic characteristics for recorded signals have been calculated: phase-time characteristics, averaging phase characteristics and averaging STFT spectrograms.

Basing on calculated basic characteristics and maps of ADC descriptor on lateral walls of transformer tanks three areas have been chosen to analyze properties of recorded signals in the point of view how propagation path of AE signal may influence on properties of recorded signals.

Analyses prove that:

a) very different structures of a recorded signal are possible even in neighbouring points of the transformer; they result from a real propagation path of $\mathrm{AE}$ impulses, generated in $\mathrm{AE}$ sources and recorded at a given measuring point (Figs. 2 and 3),

b) AE waves spreads in transformer oil are damped; it causes decrease of their amplitude and frequency band (Fig. 4),

c) particular elements of a vast source, responsible for partial discharges, may generate AE impulses simultaneously.

The work is a contribution to the method aimed at location of PD sources in the volume of power oil transformers.

\section{Acknowledgments}

The researches were financed in the frame of the project N N505 466538 of Ministry of Science and Higher Education.

\section{References}

[1] F. Witos, Z. Gacek, A. Opilski, Archiv. Acoust. 27, 65 (2002).

[2] F. Witos, Z. Gacek, P. Paduch, J. Phys. IV (France) 130, 179 (2006).

[3] F. Witos, Investigation of Partial Discharges by Means of Acoustic Emission Method and Electric Method, Silesian University od Technology, Gliwice 2008 (in Polish).

[4] F. Witos, Z. Gacek, Europ. Phys. J.-Spec. Top. 154, 239 (2008).

[5] F. Witos, Z. Gacek, Acta Phys. Pol. A 116, 422 (2009).

[6] F. Witos, A. Olszewska, Acta Phys. Pol. A 118, 1267 (2010).

[7] E. Howells, E.T. Norton, IEEE Trans. Power Apparatus Systems PAS-103, 1111 (1984).

[8] Z. Deheng, T. Kexiong, I. Xianche, in: II Conf. Properties and Application of Dielectric Mat., Vol. 2, Beijing 1988, p. 614.

[9] Y. Lu, X. Tan, X. Hu, IEEE Proc. Sci. Meas. Technol. 147, 81 (2000).

[10] J. Deng, Opt. Laser Technol. 33, 305 (2001).

[11] T. Boczar, IEEE Trans. Dielectr. Electr. Insulat. 8, 598 (2001).

[12] M. MacAlpine, Z. Zhiquiang, M.S. Demokan, Electric Power System Res. 63, 27 (2002).

[13] T. Boczar, D. Zmarzly, IEEE Trans. Dielectr. Electr. Insulat. 11, 433 (2004).

[14] S.M. Markalous, IEEE Trans. Dielectr. Electr. Insulat. 15, 278 (2008).

[15] K. Gyung-Suk, K. Il-Kwon, D. Park, S.Y. Choi, Ch.Y. Park, Curr. Appl. Phys. 9, 296 (2009). 